SYNTHESIS OF ZEOLITE LINDE F THROUGH ALKALINE TREATMENT WITH CAUSTIC POTASE FROM ROCKS OF VOLCANIC ORIGIN FROM SILLAR, PERU; ITS APPLICATION IN THE ADSORPTION OF COPPER(II)

\section{SÍNTESIS DE ZEOLITA LINDE F MEDIANTE TRATAMIENTO ALCALINO CON POTASA CAÚSTICA A PARTIR DE ROCA DE ORIGEN VOLCÁNICO PROVENIENTE DE SILLAR, PERÚ; SU APLICACIÓN EN LA ADSORCIÓN DE COBRE(II)}

Received 08132020 Accepted 12212020 Published 04302021

Vol. 38, No.1, pp. 14-25, Ene./Abr. 2021 Revista Boliviana de Química

38(1), 14-25, Jan./Apr. 2021

Bolivian Journal of Chemistry

DOI : $10.34098 / 2078-3949.38 .1 .2$

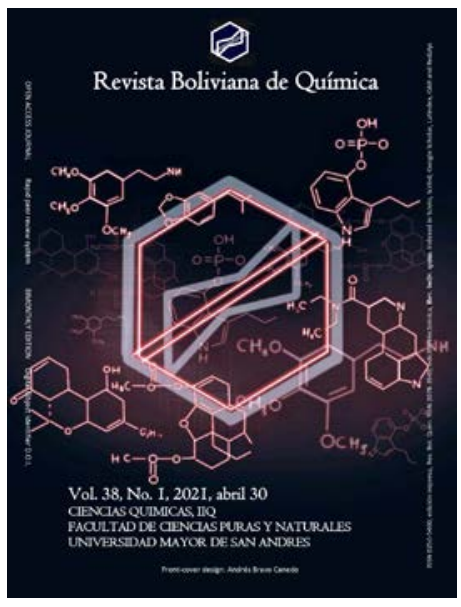

Marcelo Rodríguez Valdivia ${ }^{1, *}$, Gladys Ocharán Velásquez² ${ }^{2}$ Karen Calderón Soncco³, Víctor Benavides Paredes ${ }^{1}$

\begin{abstract}
${ }^{1}$ Escuela Profesional de Ingeniería de Materiales, Facultad de Ingeniería de Procesos FIP, Universidad Nacional San Agustín de Arequipa UNSA, Av. Independencia s/n-Pab. Ing. Materiales, phone +5154200037 , Arequipa, Perú, materiales@unsa.edu.pe, http://fip.unsa.edu.pe/ingmateriales/

${ }^{2}$ MyAP Microscopía Electrónica y Aplicaciones, Av. Rinconada del Lago 565, phone +51999453280 , Lima, Perú

${ }^{3}$ Escuela Profesional de Ingeniería Química, Facultad de Ingeniería de Procesos FIP, Universidad Nacional San Agustín de Arequipa UNSA, Av. Independencia s/n-Pab. Ing. Química, phone +51542226447 , Arequipa, Perú, iquimica@unsa.edu.pe, http://fip.unsa.edu.pe/ingquimical
\end{abstract}

Keywords: Cation exchange capacity, Volcanic material, Zeolite Linde F, Kinetics, Alkaline treatment.

Palabras clave: Capacidad de intercambio catiónico, Material volcánico, Zeolita Linde F, Cinética, Tratamiento alcalino.

\title{
ABSTRACT
}

This article reports the results of the synthesis of zeolitic material with adsorbent capacity, from rocks of volcanic origin from sillar, Arequipa, Peru. The experimentation was carried out in a stainless steel reactor using solutions with different concentrations of $\mathrm{KOH}(2.0,2.5$ and $3.0 \mathrm{M})$ at a constant temperature of $150^{\circ} \mathrm{C}$. The contact time between the volcanic material and the solution was 24,48 and 72 hours. The obtained product was characterized by means of scanning microscopic analysis (SEM) and cation exchange capacity (CEC). The results showed that synthetic zeolite was produced as a function of the concentration of $\mathrm{KOH}$ as the most influential independent 
variable. The highest cation exchange capacity (CEC) observed was $27.91 \mathrm{meq} / 100 \mathrm{~g}$ and corresponded to the formation of Linde F zeolite obtained with a 3.0 M KOH solution and for a time of 72 hours using a material whose particle size was $4 \mathrm{~mm}$. Adsorption tests were carried out using solutions containing cations $\mathrm{Cu}^{2+}$ in order to evaluate their capacity and adsorption kinetics.

\section{*Correspondent author: mrodriguezv@unsa.edu.pe}

\section{RESUMEN}

El presente artículo reporta los resultados de la síntesis de material zeolítico con capacidad adsorbente a partir de una roca de origen volcánico proveniente de sillar, Arequipa, Perú. La experimentación se efectuó en un reactor de acero inoxidable utilizando soluciones con diferentes concentraciones de $\mathrm{KOH}(2.0,2.5$ y $3.0 \mathrm{M})$, a una temperatura constante de $150^{\circ} \mathrm{C}$ y con un tiempo de contacto entre el material volcánico y la solución de 24,48 y 72 horas. El producto obtenido se caracterizó mediante, análisis microscópico de barrido (SEM) y capacidad de intercambio catiónico (CIC).

Los resultados demostraron que se produjo zeolita sintética en función de la concentración de $\mathrm{KOH}$ como variable independiente más influyente. La mayor capacidad de intercambio catiónico (CIC) observada fue de 27.91 meq/100g y correspondió a la formación de zeolita Linde F obtenida con una solución de KOH 3.0 M y por un tiempo de 72 horas usando un material cuyo tamaño de partícula fue de $4 \mathrm{~mm}$. Se realizaron pruebas de adsorción utilizando soluciones conteniendo cationes $\mathrm{Cu}^{2+}$ a fin de evaluar su capacidad y cinética de adsorción.

\section{INTRODUCCIÓN}

El sur del Perú presenta yacimientos importantes de minerales no metálicos, uno de los más conocidos es sillar (Arequipa, Peru) con reservas de roca volcánica o mineral ignimbrita. Industrias como la minería, la agricultura, la alimentación animal, la potabilización del agua, la industria farmacéutica, etc. [1], requieren de materiales adsorbentes de bajo costo y con capacidad de ser utilizados en remediación ambiental. Una alternativa viable es la utilización de las zeolitas naturales o sintéticas, pero que exhiban altas capacidades de intercambio catiónico. Es posible sintetizar zeolitas a partir de reactivos químicos puros y de precursores de origen natural (zeolita natural, perlita, bentonita, caolín, puzolana, cenizas volantes, etc.), ya que son abundantes en la naturaleza y contienen un alto contenido de sílice y alúmina [2]. Las zeolitas encuentran su aplicación debido a su eficiente capacidad de actuar como intercambiadores de cationes, catalizadores, filtros, y biofiltros, en diversos procesos químicos y de tratamiento del medio ambiente.

Métodos como la conversión hidrotermal, fusión alcalina y conversión hidrotermal, conversión asistida de por micro ondas [3,4]; han sido desarrollados empleando especialmente cenizas volantes provenientes de centrales termoeléctricas como precursores baratos [5]. Los tipos de zeolitas sintéticas que se forman presentan diversas propiedades físicas, químicas, mineralógicas y morfológicas de acuerdo a las diferentes condiciones de obtención como la temperatura, alcalinidad, cristalización y relación líquido/sólido. En consecuencia, un control de todas estas variables en el proceso de síntesis puede resultar en la obtención de un producto zeolítico adecuado para su aplicación industrial [6].

De acuerdo a estos antecedentes, se considera oportuno realizar el presente trabajo de investigación a fin de dar valor agregado al mineral sillar que existe en la región Arequipa, Perú, aplicando un tratamiento de conversión alcalina con el fin de obtener materiales zeolíticos con capacidad de intercambiar cationes. Se busca además evaluar sus posibles aplicaciones en el campo de la remediación ambiental para el tratamiento de efluentes mineros o industriales.

El presente estudio se ha dividido en dos etapas. En la primera, el sillar fue caracterizado mediante análisis químicos y físicos. Luego se aplicó el tratamiento alcalino con soluciones de $\mathrm{KOH}$ variando el tamaño de partícula, la concentración y el tiempo de contacto. El producto zeolítico obtenido fue analizado mediante análisis químicomorfológico al microscopio electrónico (SEM) y la capacidad de intercambio catiónico (CIC). En la segunda etapa, se realizaron pruebas de adsorción utilizando soluciones conteniendo $\mathrm{Cu}^{2+}$. Los resultados obtenidos, los cuales son consistentes con los reportados en la literatura, permiten avisorar positivamente la posibilidad de profundizar las consideraciones técnico-económicas relativas a la zeolitización del mineral ignimbrítico sillar para obtener zeolita sintética.

\footnotetext{
Downloadable from: Revista Boliviana 


\section{Materiales y métodos}

\section{Preparación del Material}

Sobre el material volcánico (sillar) se realizaron operaciones de chancado y tamizado para obtener tamaños de partícula de 2, 3 y 4 mm.; las cuales han sido empleadas para las pruebas de síntesis con tratamiento alcalino. Se eligieron estas clases granulométricas con el propósito explorar la posibilidad de su potencial uso en la producción de filtros para el tratamiento de agua contaminada.

\section{Equipos de Caracterización y análisis}

La composición química y el análisis morfológico del material volcánico y del producto zeolítico, se analizó empleando un Microscopio Electrónico (SEM) modelo FEI Quanta 200 de alto y bajo vacío con detectores de microanálisis SED y BSED [7]. Para la evaluación de la capacidad de intercambio catiónico (CIC) se realizaron pruebas de doble intercambio catiónico usando soluciones acetato de sodio $1.0 \mathrm{~N}$ y acetato de amonio $1.0 \mathrm{~N}$. El análisis de máxima adsorción de $\mathrm{Na}^{+}$y de $\mathrm{Cu}^{2+}$, fue posible utilizando un equipo de adsorción Perkin Elmer OPTIMA-2100-DV ICP en laboratorio de análisis Laboratorios del Sur.

\section{Reactivos}

Las soluciones empleadas fueron preparadas con las siguientes sales: $\mathrm{KOH}$ (99.5\%), $\mathrm{NaC}_{2} \mathrm{H}_{3} \mathrm{O}_{2} \cdot 3 \mathrm{H}_{2} \mathrm{O}$, $\mathrm{NH}_{4} \mathrm{C}_{2} \mathrm{H}_{3} \mathrm{O}_{2} .3 \mathrm{H}_{2} \mathrm{O}$ (99.0\%), $\mathrm{CuCl}_{2} .2 \mathrm{H}_{2} \mathrm{O}$ (99.09\%), alcohol isopropílico (99.0\%), adquiridos de la empresa Diproquim.

\section{Pruebas para la obtención de zeolita Linde F}

Las pruebas de obtención de zeolita sintética se realizaron empleando un recipiente de acero inoxidable AISI 304 que fue colocado en el interior de horno a una temperatura de $150^{\circ} \mathrm{C}$. En el recipiente se colocaron $10 \mathrm{~g}$ de material volcánico con tamaños de partícula de 2, 3 y $4 \mathrm{~mm}$ y soluciones de $\mathrm{KOH}$ con concentraciones de 2, 2.5 y $3 \mathrm{M}$, manteniendo una relación sólido/líquido de 12.5. El tiempo de contacto entre el entre el líquido y el sólido fue de 24, 48 y 72 horas. Singh, Ah., et. al. [8], reporta haber empleado $150^{\circ} \mathrm{C}$ y 48 horas para sintetizar zeolita por activación alcalina utilizando soluciones de $\mathrm{KOH}$. El producto obtenido fue lavado con agua destilada y filtrado para finalmente llevarlo a secado en estufa a $60^{\circ} \mathrm{C}$ por un tiempo de 6 horas, condiciones definidas de acuerdo a la literatura revisada [9].

\section{Análisis de la capacidad de intercambio catiónico (CIC)}

Se empleó el Método analítico 9081 de determinación de la capacidad de intercambio catiónico [10]:

Pesar $4 \mathrm{~g}$ de material de tamaño de partícula fina, y transferir la muestra hacia el contenedor para centrifugación de base circular con una capacidad de $50 \mathrm{~mL}$.

a) Añadir $33 \mathrm{ml}$ de $1.0 \mathrm{~N}$ de la solución de acetato de sodio, tapar el tubo, someter a agitador mecánico por $5 \mathrm{~min}$, y centrifugar la solución hasta que el líquido este limpio o claro.

b) Decantar el líquido y repetir el párrafo (a), 3 veces más.

c) Añadir $33 \mathrm{ml}$ de alcohol isopropílico (99\%), tapar el tubo, someter a agitador mecánico por 5 min, y centrifugar hasta que el líquido esté claro.

d) Repetir el procedimiento del párrafo (c), 2 veces más.

e) Añadir $33 \mathrm{~mL}$ de la solución acetato de amonio, tapar el tubo, someter a agitador mecánico por 5 min, y centrifugar hasta que el líquido supernadante esté claro. Decantar la solución dentro de un frasco con capacidad para $100 \mathrm{~mL}$.

f) Repetir el procedimiento descrito en el párrafo (e), 2 veces más.

g) Completar un volumen de $100 \mathrm{~mL}$ solución de acetato de amonio y determinar la concentración de $\mathrm{Na}^{+}$por absorción atómica, emisión espectroscópica, o un método equivalente.

\section{Pruebas de adsorción de $\mathrm{Cu}^{2+}$}

A fin de evaluar la capacidad de adsorción en función del tiempo, se realizaron pruebas con el producto zeolítico que presentó la más alta capacidad de intercambio catiónico. En botellas de vidrio conteniendo $1 \mathrm{~g}$ de zeolita se echaron 
$150 \mathrm{~mL}$ de solución $\mathrm{Cu}^{2+}$ con una concentración de $1000 \mathrm{mg} / \mathrm{L}$. Después de haber transcurrido 24, 48 y 72 horas se tomaron $100 \mathrm{~mL}$ de solución para análisis de $\mathrm{Cu}^{2+}$.

La determinación de la capacidad de adsorción a diferentes concentraciones de $\mathrm{Cu}^{2+}$, se llevó a cabo empleando recipientes de vidrio de $200 \mathrm{~mL}$ de capacidad, donde se colocaron $150 \mathrm{~mL}$ de solución de solución de $\mathrm{Cu}^{2+}$ con concentraciones de 1092, 871, 764, 327, 109 y 55 mg/L y 1g de producto zeolítico. Durante las pruebas se agitó los recipientes para mejorar el contacto entre la solución y el material zeolítico. El tiempo de contacto será determinado por las pruebas a tiempo variable. Luego de transcurrido el tiempo de contacto entre el sólido y el líquido, se filtraron las soluciones y se tomaron $100 \mathrm{~mL}$ de solución para analizar $\mathrm{Cu}^{2+}$ mediante adsorción atómica.

\section{RESULTADOS Y DISCUSIÓN}

\section{Caracterización del material volcánico (sillar)}

\section{Composición química}

La Tabla No. 1, presenta el análisis del material volcánico (sillar) en su estado natural.

Tabla No. 1. Composición Química - Sillar en estado natural

\begin{tabular}{cccccccc}
\hline Elemento & $\mathbf{N a}_{2} \mathbf{O}$ & $\mathbf{M g O}$ & $\mathbf{A l}_{2} \mathbf{O}_{3}$ & $\mathbf{S i O}_{2}$ & $\mathbf{K}_{2} \mathbf{O}$ & $\mathbf{C a O}$ & $\mathbf{F e}_{2} \mathbf{O}_{3}$ \\
\hline Composición \% & $4.92 \%$ & $0.27 \%$ & $14.43 \%$ & $74.99 \%$ & $3.02 \%$ & $1.11 \%$ & $1.11 \%$ \\
\hline
\end{tabular}

Se puede apreciar claramente un alto contenido en $\mathrm{SiO}_{2}$ y $\mathrm{Al}_{2} \mathrm{O}_{3}$ esenciales para el proceso de síntesis de zeolita mediante tratamiento alcalino. Así, se tiene que el sillar presenta un contenido de $\mathrm{SiO}_{2}+\mathrm{Al}_{2} \mathrm{O}_{3}$ igual a $89.42 \%$. Ferrarini, S., et. al. [11], indican que esta relación debe ser mayor a 89\%, además de que estos elementos se encuentran presentes en minerales como el cuarzo $\left(\mathrm{SiO}_{2}\right)$ y mullita $\left(3 \mathrm{Al}_{2} \mathrm{O}_{3} \cdot 2 \mathrm{SiO}_{2}\right)$ y los compuestos que contienen Ca, Fe son impurezas que pueden limitar su aplicación en la síntesis de zeolitas.

\section{Caracterización morfológica}

La Figura No. 1, muestra formaciones piroclásticas de aspecto vítreo que contienen $\mathrm{SiO}_{2}$ y $\mathrm{Al}_{2} \mathrm{O}_{3}$ (cristobalita, cuarzo, anortoclasa y ortoclasa) además de porosidad de diverso tamaño.

\section{Síntesis de zeolita Linde $F$}

La Tabla No. 2, muestra los valores de la capacidad de intercambio catiónico (CIC) determinados en muestras de los productos zeolíticos obtenidos en diferentes condiciones de prueba, y la presentada por el material sin tratamiento.

La Tabla No. 3, presenta el contenido zeolítico comparado con una zeolita Linde F obtenida por síntesis a partir de fly-ash con una capacidad de intercambio catiónico igual a 140 meq/100g [12]. Se demuestra que el producto obtenido en esta investigación presenta un contenido máximo de zeolita Linde F de 19.93\%.

Tabla No. 2. Capacidad de intercambio catiónico (CIC)

\begin{tabular}{ccc}
\hline Prueba & Condiciones de Prueba & (CIC) meq/100g \\
\hline 1 & $2 \mathrm{~mm} / 2 \mathrm{M} / 24 \mathrm{~h}$ & 10.96 \\
2 & $4 \mathrm{~mm} / 2 \mathrm{M} / 24 \mathrm{~h}$ & 14.26 \\
3 & $2 \mathrm{~mm} / 3 \mathrm{M} / 24 \mathrm{~h}$ & 27.13 \\
4 & $4 \mathrm{~mm} / 3 \mathrm{M} / 24 \mathrm{~h}$ & 16.00 \\
5 & $2 \mathrm{~mm} / 2 \mathrm{M} / 72 \mathrm{~h}$ & 11.91 \\
6 & $4 \mathrm{~mm} / 2 \mathrm{M} / 72 \mathrm{~h}$ & 11.39 \\
7 & $2 \mathrm{~mm} / 3 \mathrm{M} / 72 \mathrm{~h}$ & 27.04 \\
8 & $4 \mathrm{~mm} / 3 \mathrm{M} / 72 \mathrm{~h}$ & 27.91 \\
9 & $3 \mathrm{~mm} / 2.5 \mathrm{M} / 48 \mathrm{~h}$ & 11.65 \\
10 & $3 \mathrm{~mm} / 2.5 \mathrm{M} / 48 \mathrm{~h}$ & 12.26 \\
11 & $3 \mathrm{~mm} / 2.5 \mathrm{M} / 48 \mathrm{~h}$ & 11.13 \\
12 & Sillar & 1.83 \\
\hline
\end{tabular}




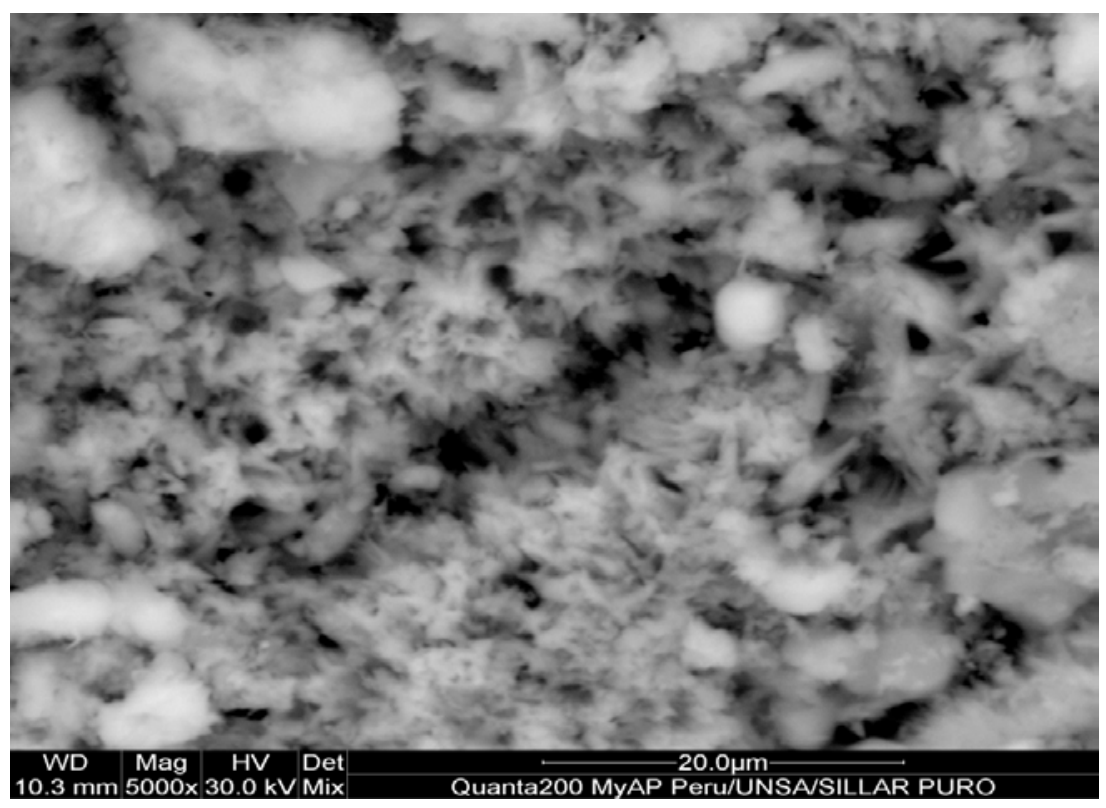

Figura No. 1. Micrografía al SEM mostrando formaciones piroclásticas

Los resultados obtenidos manifiestan altos valores de capacidad de intercambio catiónico, del orden de 27.91 y 27.13 meq/100g. Es posible concluir que a una concentración de KOH de 3 M existe una alta disolución de la sílice y la alúmina durante el proceso de conversión por tratamiento alcalino.

Tabla No. 3. Contenido de zeolita Linde F para diferentes condiciones de prueba

\begin{tabular}{cccccc}
\hline Muestra & $\begin{array}{c}\text { Tamaño de partícula } \\
(\mathrm{mm})\end{array}$ & $\begin{array}{c}\text { Concentración KOH } \\
(\mathrm{M})\end{array}$ & $\begin{array}{c}\text { Tiempo } \\
(\text { Horas })\end{array}$ & $\begin{array}{c}\text { CIC } \\
(\mathrm{meq} / 100 \mathrm{~g})\end{array}$ & $\begin{array}{c}\text { Zeolita Linde F } \\
(\%)\end{array}$ \\
\hline 1 & 2 & 2 & 24 & 11.0 & 7.86 \\
2 & 4 & 2 & 24 & 14.3 & 10.21 \\
3 & 2 & 3 & 24 & 27.1 & 19.36 \\
4 & 4 & 3 & 24 & 16.0 & 11.43 \\
5 & 2 & 2 & 72 & 11.9 & 8.50 \\
6 & 4 & 2 & 72 & 11.4 & 8.14 \\
7 & 2 & 3 & 72 & 27.0 & 19.29 \\
8 & 4 & 3 & 72 & 27.9 & 19.93 \\
9 & 3 & 2.5 & 48 & 11.7 & 8.36 \\
10 & 3 & 2.5 & 48 & 12.3 & 8.79 \\
11 & 3 & 2.5 & 48 & 11.1 & 7.93 \\
\hline
\end{tabular}

\section{Caracterización del producto zeolítico}

La zeolita sintética que mostró la mayor capacidad de intercambio catiónico (4mm/3M/72horas) fue caracterizada física, química y morfológicamente para definir su tipo e identificarla.

\section{Composición química}

La Tabla No. 4, muestra el resultado del análisis químico expresado en óxidos presentes. 


\begin{tabular}{cccccccc}
\hline Elemento & $\mathbf{N a}_{2} \mathbf{O}$ & $\mathbf{A l}_{2} \mathbf{O}_{3}$ & $\mathbf{S i O}_{2}$ & $\mathbf{K}_{\mathbf{2}} \mathbf{O}$ & $\mathbf{C a O}$ & $\mathbf{T i O}_{2}$ & $\mathbf{F e}_{2} \mathbf{O}_{3}$ \\
\hline Composición \% & $6.33 \%$ & $20.34 \%$ & $61.52 \%$ & $7.28 \%$ & $1.75 \%$ & $0.54 \%$ & $2.23 \%$ \\
\hline
\end{tabular}

\section{Caracterización morfológica}

La Figura No. 2, muestran formaciones de cristales tetragonales y prismáticos de zeolita Linde F, a partir de la disolución de la matriz sillar. Miyaji, F., et. al [12], mencionan la obtención de cristales con forma de prismas regulares en la síntesis de zeolita linde F empleando fly-ash.

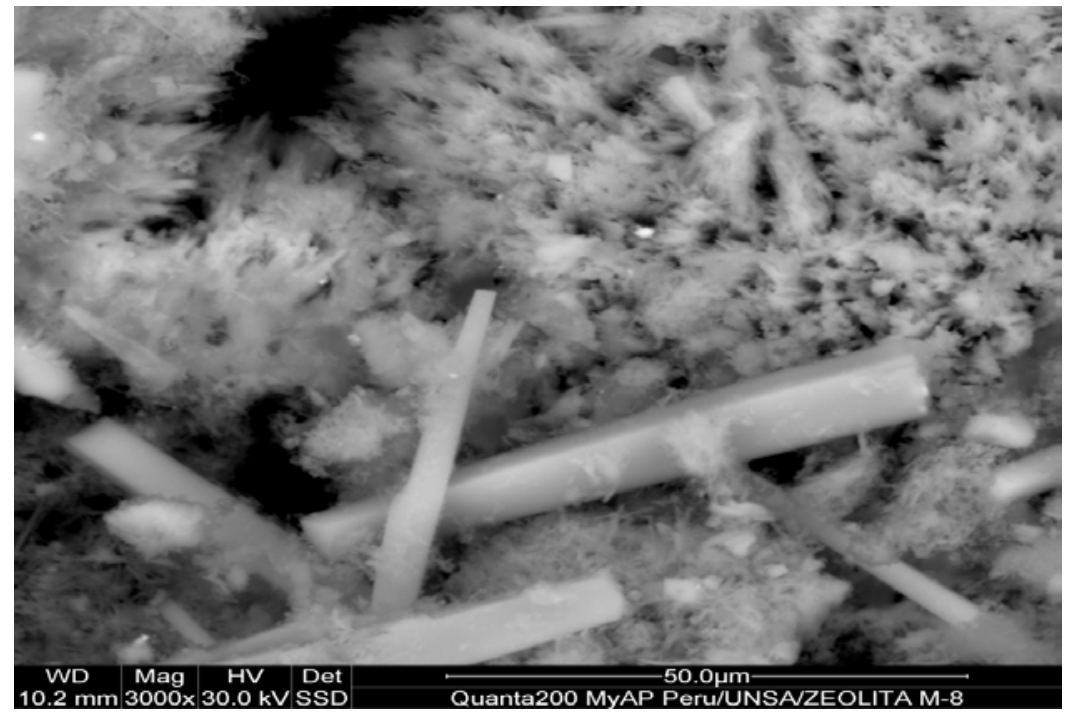

Figura No. 2. Formación de zeolita Linde F en matriz piroclástica del sillar. Prueba 4mm/3M/72h.

Comparando los análisis químicos del sillar al natural y la que corresponde a la zeolita sintética Linde F por medio del software TRIPLOT Versión 4.1.2, es posible apreciar el movimiento de la composición de la zeolita en función de sus principales componentes químicos $\mathrm{SiO}_{2}, \mathrm{Al}_{2} \mathrm{O}_{3}$ y $\mathrm{K}_{2} \mathrm{O}$. La Figura No. 3, muestra el diagrama ternario conteniendo las composiciones del sillar y del material zeolítico Linde $\mathrm{F}$. Es notorio el enriquecimiento de $\mathrm{K}_{2} \mathrm{O}$ y $\mathrm{Al}_{2} \mathrm{O}_{3}$ de 3.02 a $7.28 \%$ y de 14.43 a $20.34 \%$ respectivamente.

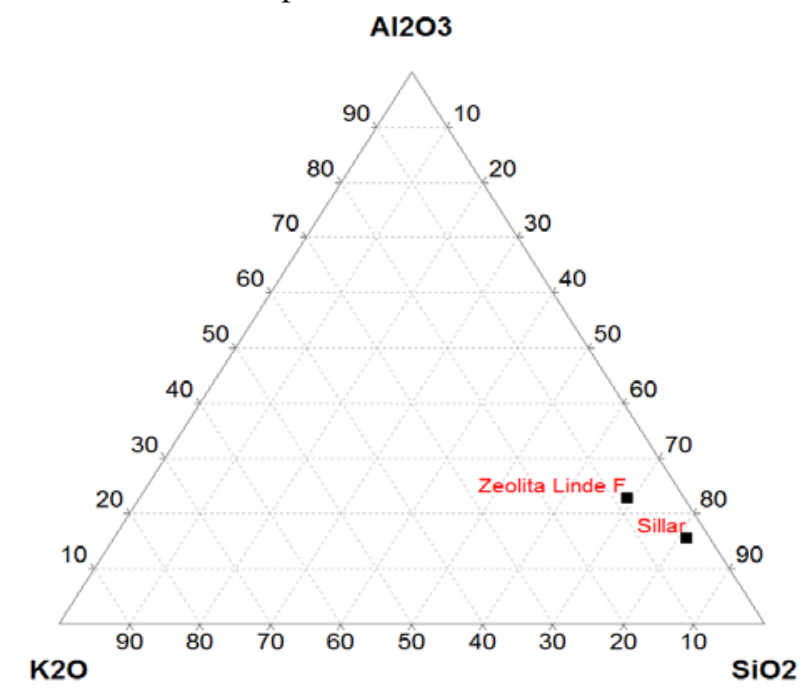

Figura No.3. Diagrama ternario para componentes $\mathrm{SiO}_{2}, \mathrm{Al}_{2} \mathrm{O}_{3}$ y $\mathrm{K}_{2} \mathrm{O}$ 


\section{Adsorción del catión $\mathrm{Cu}^{2+}$}

La cantidad de catión adsorbido fue calculada como la diferencia entre la concentración inicial y final en las soluciones [13,14]. La Tabla No. 5 muestra la capacidad de adsorción de $\mathrm{Cu}^{2+}$ en función del tiempo de contacto.

Tabla No. 5. Adsorción de cationes $\mathrm{Cu}^{2+}$ con Zeolita Linde F

\begin{tabular}{|c|c|c|c|c|c|c|c|}
\hline $\begin{array}{l}\text { Tiempo } \\
\text { (horas) }\end{array}$ & $\begin{array}{l}\text { Conc. inicial } \\
\text { (mg/L) }\end{array}$ & $\begin{array}{l}\text { Conc. final } \\
\text { (mg/L) }\end{array}$ & $\begin{array}{c}\mathrm{Cu}^{2+} \text { inicial } \\
(\mathrm{mg})\end{array}$ & $\begin{array}{l}\mathrm{Cu}^{2+} \text { final } \\
\quad(\mathrm{mg})\end{array}$ & $\begin{array}{c}\mathrm{Cu}^{2+} \\
\text { adsorbido } \\
\text { (mg) }\end{array}$ & $\begin{array}{c}\mathrm{Cu}^{2+} \\
\text { adsorbido } \\
(\mathrm{mg} / \mathrm{g})\end{array}$ & $\begin{array}{c}\mathrm{Cu}^{2+} \\
\text { adsorbido } \\
(\%)\end{array}$ \\
\hline Alimentación & 1000 & & & & & & \\
\hline 4 & & 916.00 & 150.0 & 91.6 & 58.4 & 58.4 & 38.9 \\
\hline 8 & & 916.00 & 150.0 & 91.6 & 58.4 & 58.4 & 38.9 \\
\hline 12 & & 916.00 & 150.0 & 91.6 & 58.4 & 58.4 & 38.9 \\
\hline 16 & & 896.00 & 150.0 & 89.6 & 60.4 & 60.4 & 40.3 \\
\hline
\end{tabular}

De la Tabla No. 5, se deriva claramente que después de 8 horas, para estas condiciones de ensayo, la cantidad, en mg de $\mathrm{Cu}^{2+}$ por gramo de zeolita, de los cationes intercambiados permanece constante.

\section{Modelo cinético de adsorción}

El modelo propuesto por Blanchard [15], denominado de pseudo-segundo orden resulta ser el más adecuado para explicar el procedimiento de adsorción del catión $\mathrm{Cu}^{2+}$ sobre la zeolita sintética. Modelo cinético pseudo-segundo orden (1):

$$
\frac{\mathrm{t}}{\mathrm{q}}=\frac{1}{\mathrm{k} 2 \mathrm{qe}^{2}}+\frac{\mathrm{t}}{\mathrm{qe}}
$$

Donde, "qe" es la cantidad de $\mathrm{Cu}^{2+}$ adsorbido en el equilibrio por la zeolita expresado en (mg/g), "q" es la cantidad de $\mathrm{Cu}^{2+}$ en un tiempo $\mathrm{t}$ en $(\mathrm{mg} / \mathrm{g}), \mathrm{k}_{2}$ es la constante del modelo de pseudo-segundo orden y $\mathrm{t}$ es el tiempo de contacto. La Figura No. 4, presenta la cinética de adsorción para el modelo linealizado de pseudo-segundo orden.

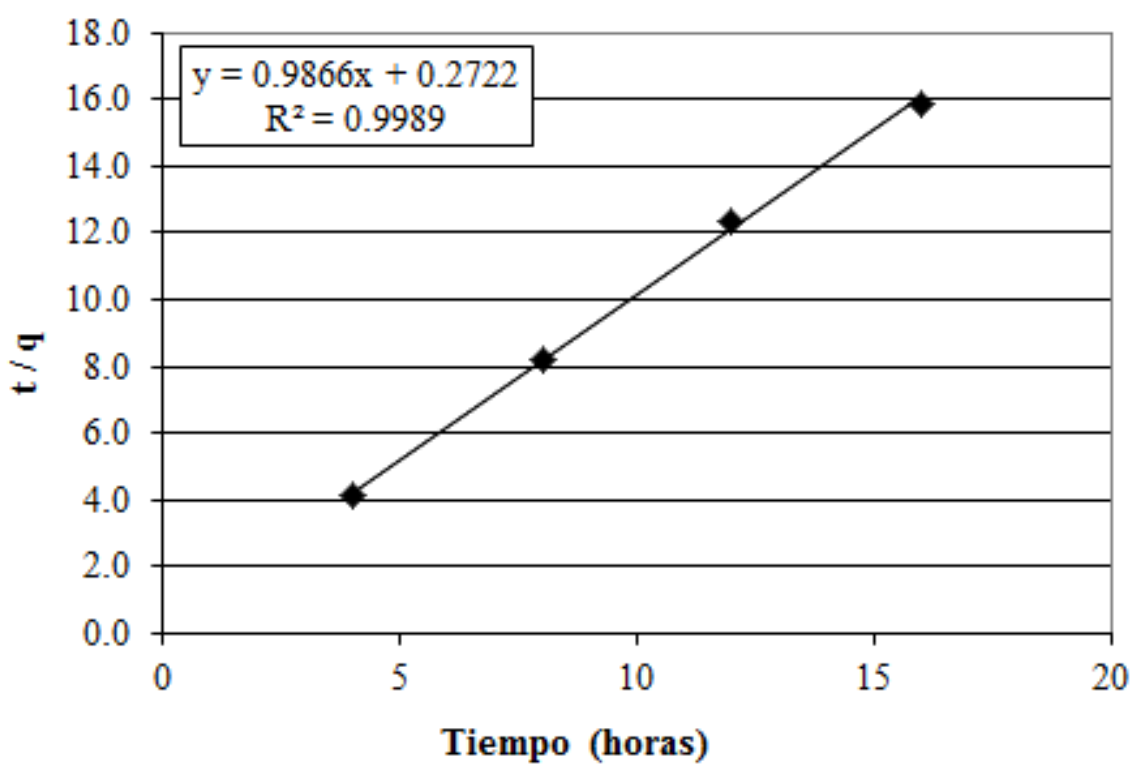

Figura No. 4. Cinética de adsorción de $\mathrm{Cu}^{2+}$ para el modelo de pseudo-segundo orden

La Tabla No. 6 muestra los resultados de las pruebas de adsorción de $\mathrm{Cu}^{2+}$ a concentración variable y un tiempo de contacto constante de 8 horas. 
Tabla No. 6. Adsorción de $\mathrm{Cu}^{2+}$ a diferentes concentraciones

\begin{tabular}{cccccccc}
\hline Prueba & $\begin{array}{c}\text { Conc.Inicial } \\
(\mathrm{mg} / \mathrm{L})\end{array}$ & $\begin{array}{c}\text { Análisis } \\
(\mathrm{mg} / \mathrm{L})\end{array}$ & $\begin{array}{c}\mathrm{Cu}^{2+} \\
\text { inicial(mg) }\end{array}$ & $\begin{array}{c}\mathrm{Cu}^{2+} \\
\text { final(mg) }\end{array}$ & $\begin{array}{c}\mathrm{Cu}^{2+} \\
\text { adsorbido } \\
(\mathrm{mg})\end{array}$ & $\begin{array}{c}\mathrm{Cu}^{2+} \\
\text { adsorbido } \\
(\mathrm{mg} / \mathrm{g})\end{array}$ & $\begin{array}{c}\mathrm{Cu}^{2+} \\
\text { adsorbido } \\
(\%)\end{array}$ \\
\hline 1 & 1092.00 & 896.00 & 163.80 & 134.40 & 29.40 & 29.40 & 17.95 \\
2 & 870.99 & 720.00 & 131.04 & 108.32 & 22.72 & 22.72 & 17.34 \\
3 & 764.40 & 624.00 & 114.66 & 93.60 & 21.06 & 21.06 & 18.37 \\
4 & 326.58 & 266.40 & 49.14 & 40.09 & 9.05 & 9.05 & 18.43 \\
5 & 108.97 & 78.10 & 16.38 & 11.74 & 4.64 & 4.64 & 28.33 \\
6 & 54.57 & 42.10 & 8.19 & 6.32 & 1.87 & 1.87 & 22.85 \\
\hline
\end{tabular}

\section{Isotermas de Adsorción de Freundlich, Langmuir y Temkin}

Los datos experimentales fueron correlacionados usando los modelos de adsorción propuestos por Freundlich, Lagmuir y Temkin $[16,17]$.

La ecuación linealizada de Freundlich, es la siguiente (2):

$$
\log q e=\log K f+\frac{1}{n} \log C e
$$

Donde, $C_{e}$ es la concentración en equilibrio de la solución a adsorber $(\mathrm{mg} / \mathrm{L}), q_{e}$ es la capacidad de adsorción del adsorbato, en (mg/g), $\mathrm{K}_{\mathrm{f}}$ es la constante de Freundlich relacionada con la capacidad de adsorción, $\mathrm{n}$ es la constante de Freundlich relacionada con la intensidad de la adsorción o grado de favorabilidad de adsorción.

La ecuación linealizada de Langmuir es la siguiente (3):

$$
\frac{1}{q e}=\left(\frac{1}{\text { Kl xqmáx }}\right) \frac{1}{C e}+\frac{1}{q m a ́ x}
$$

Donde, q qáx. es la capacidad máxima de adsorción del colorante bajo las condiciones dadas (mg/g), $\mathrm{Kl}$ es la constante de Langmuir que indica la afinidad del adsorbato por el adsorbente, $q_{\mathrm{e}}$ es la capacidad de adsorción del adsorbato, en (mg/g), $C_{\mathrm{e}}$ es la concentración de la solución en el equilibrio $(\mathrm{mg} / \mathrm{L})$.

La ecuación linealizada de Tenkim, se expresa de la siguiente forma (4):

$$
q e=B t x \ln (A t)+B t x \ln (C e)
$$

Donde, qe es la capacidad de adsorción del adsorbato, en (mg/g), Bt es la constante que relaciona el calor de adsorción (J/mol), At es la constante de unión de equilibrio (máxima energía de unión) (L/mg), $C_{e}$ es la concentración en equilibrio de la solución a adsorber $(\mathrm{mg} / \mathrm{L})$.

Las Figuras No. 5, 6 y 7 muestran las isotermas de adsorción de Freundlich, Langmuir y Tenkim.

La Tabla No. 7 muestra los valores de las constantes relativas a las isotermas de Freundlich, Langmuir y Tenkim con sus correspondientes coeficientes de correlación (R) relativos a los valores experimentales al equilibrio.

$\mathrm{El}$ análisis de los resultados obtenidos en las pruebas de síntesis para obtener zeolita Linde $\mathrm{F}$ a partir del sillar y las pruebas de adsorción de $\mathrm{Cu}^{2+}$ por el producto zeolítico, revelaron que: a la temperatura de $150^{\circ} \mathrm{C}$, y con una condición de prueba 4mm/3M/72 horas, la parte vítrea contenida en el sillar se disuelve y pasa a formar cristales de zeolita Linde F sobre ella. A esta temperatura se asegura la disolución de los constituyentes del material inicial y la formación de cristales de zeolita.

\section{Efecto del tamaño de partícula.}

Para tamaños de partícula de 2 y $4 \mathrm{~mm}$, con alta concentración y con tiempos prolongados de contacto entre la solución y el sólido, la capacidad de intercambio catiónico alcanza entre 27.04 y $27.91 \mathrm{meq} / 100 \mathrm{~g}$. Debido a que se tiene partículas de tamaño grande el proceso de conversión se realiza sólo a nivel de la superficie.

$$
\text { Downloadable from: Revista Boliviana } 21 \text { de Química. Volumen } 38 \text { №1. Año } 2020
$$




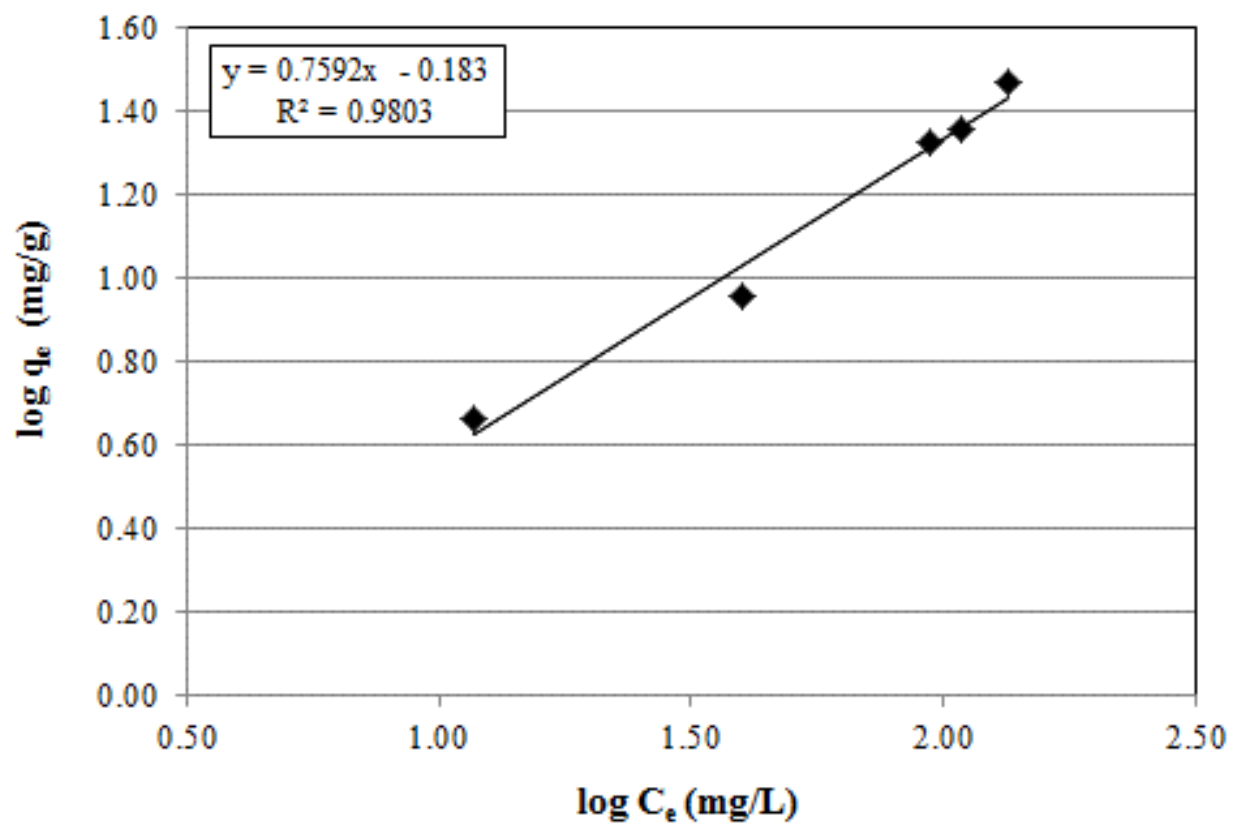

Figura No. 5. Isoterma de adsorción de Freundlich

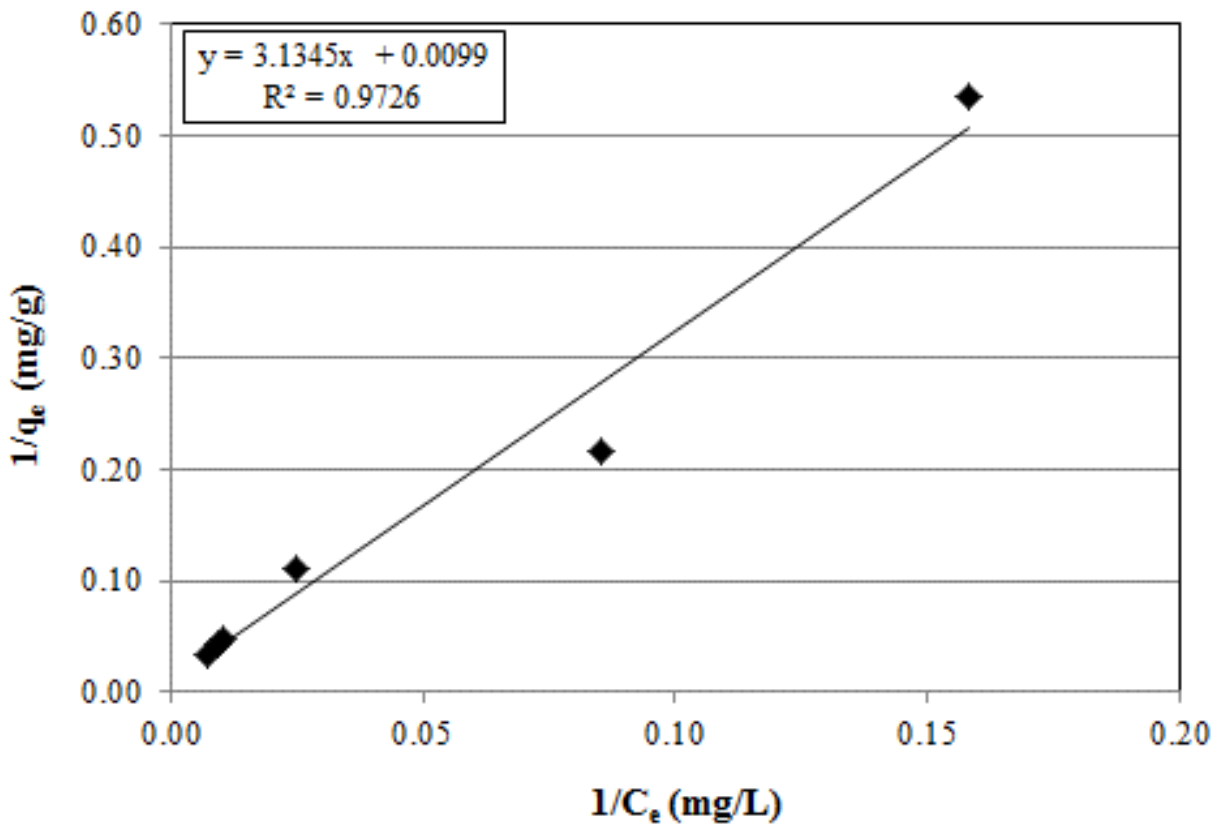

Figura No. 6. Isoterma de adsorción de Langmuir 


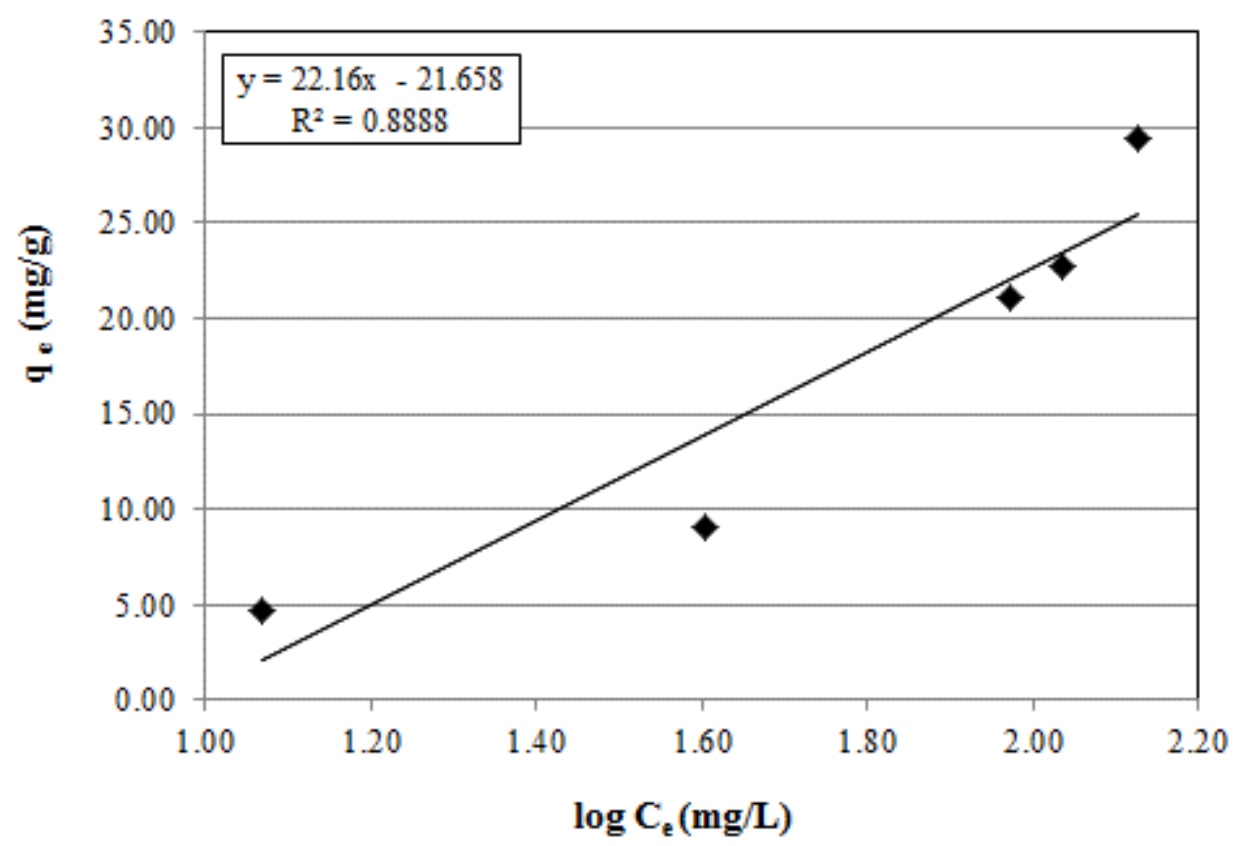

Figura No. 7. Isoterma de adsorción de Tenkim

Tabla No. 7. Valores de parámetros de las isotermas Langmuir, Freundlich y Temkin y su correspondiente coeficiente de correlación (R).

\begin{tabular}{cccc}
\hline ISOTERMA & CONSTANTES & VALOR & $\mathbf{R}^{2}$ \\
\hline \multirow{2}{*}{ Langmuir } & $\mathrm{K}_{\mathrm{L}}(\mathrm{L} / \mathrm{mg})$ & 0.00316 & 0.972 \\
& $\mathrm{qmáx}^{(\mathrm{mg} / \mathrm{g})}$ & 101.01 & \\
\hline \multirow{2}{*}{ Freundlich } & $\mathrm{K}_{\mathrm{f}}(\mathrm{L} / \mathrm{g})$ & 1.524 & 0.980 \\
& $\mathrm{n}$ & 1.3172 & \\
\hline \multirow{2}{*}{ Temkin } & $\mathrm{Bt}(\mathrm{J} / \mathrm{mol})$ & 22.160 & \multirow{2}{*}{0.888} \\
& $\mathrm{At}(\mathrm{L} / \mathrm{mg})$ & 0.376 & \\
\hline
\end{tabular}

\section{Efecto de la concentración de $\mathrm{KOH}$}

Con respecto a la concentración de la solución de $\mathrm{KOH}$, los mejores resultados de la CIC se dan cuando se emplean concentraciones de 3 M (27.04, 27.13 y 27.91 meq/100g). A mayor concentración, el pH se incrementa, lo que asegura una buena disolución de las fases minerológicas que contienen silicio y aluminio para formar un gel que luego cristalizará en productos zeolíticos. Hu, T., et. al., [18] afirma que la alcalinidad en la mezcla es un factor importante a considerar en la determinación de la cristalinidad y composición del producto zeolítico.

\section{Efecto del tiempo de contacto}

Los mejores resultados se obtienen en pruebas con mayores tiempos de conversión. De manera particular, para tiempos de conversión de 72 horas y tamaños de partículas de 2 y $4 \mathrm{~mm}$ se consiguieron los mejores valores en la CIC cuando se emplearon altas concentraciones de KOH. Chen, C. and Cheng, T., [19], sostienen que es importante considerar una dependencia entre el tiempo y la temperatura de reacción, así se tiene que a una temperatura alta y a un tiempo prolongado, se asegura la presencia de sílicio y aluminio libre provenientes de la disolución de la fase vítrea del precursor. 
La adsorción del material zeolítico en miligramo por gramo para el catión $\mathrm{Cu}^{2+}$ es del orden de $29.40 \mathrm{mg} / \mathrm{g}(17.95 \%)$ para una concentración inicial de $1092.00 \mathrm{mg} / \mathrm{L}$, y de $4.64 \mathrm{mg} / \mathrm{g}$ (28.33\%) para una concentración inicial de 108.97. Este comportamiento depende del hecho de que el fenómeno de adsorción es influenciado por la densidad de carga, así como por la energía de hidratación y el radio atómico de los cationes $\mathrm{Cu}^{2+}$. El ajuste del modelo cinético de pseudo-segundo orden $\left(\mathrm{R}^{2}=0.999\right)$ permite afirmar que la adsorción de los iones $\mathrm{Cu}^{2+}$ ocurre sobre los sitios activos de la zeolita e involucra mecanismos de quimisorción entre el adsorbato y el adsorbente [20,21].

El análisis de las isotermas de Langmuir, Freundlich y Temkin, hacen posible evidenciar que existe una linealidad y ajuste más cercano con la isoterma de Freundlich $\left(\mathrm{R}^{2}=0.980\right)$. Por lo tanto, se puede considerar que esta isoterma es la que rige el proceso de adsorción de iones $\mathrm{Cu}^{2+}$ sobre la zeolita Linde $\mathrm{F}$. Los centros activos del adsorbente son energéticamente heterogéneos, lo que indica que presentan diferentes afinidades por lo que inicialmente el fenómeno de adsorción se origina porque los sitios activos con mayor afinidad son ocupados por el catión $\mathrm{Cu}^{2+}$ y se genera la formación de una capa, sin embargo, los sitios libres restantes interactúan con el adsorbato por medio de las fuerzas de Van der Waals dando lugar a la formación de otras capas (multicapas) [22,23]. El valor de la constante $1 / n$ de 0.759 , evidencia que el fenómeno de adsorción de $\mathrm{Cu}^{2+}$ sobre la zeolita Linde $\mathrm{F}$ es favorable.

\section{CONCLUSIONES}

El tratamiento alcalino en diferentes condiciones del mineral de origen volcánico del sillar proveniente de las canteras de la ciudad de Arequipa-Perú, produce zeolita Linde F con características texturales y de capacidad de intercambio catiónico propias. El producto zeolítico obtenido en las condiciones de prueba empleando tamaños de partícula de $4 \mathrm{~mm}$, solución de KOH $3 \mathrm{M}$ y un tratamiento de síntesis por 72 horas a una temperatura constante de $150^{\circ} \mathrm{C}$, presenta una capacidad de intercambio catiónico $\mathrm{CIC}=27.91 \mathrm{meq} / 100 \mathrm{~g}$. Bajo estas condiciones experimentales, se forma zeolita sintética Linde $\mathrm{F}$ en un contenido de 19.9\%. Empleando el material zeolítico obtenido a la condición de prueba $4 \mathrm{~mm} / 3 \mathrm{M} / 72$ horas; se tiene una adsorción de $29.40 \mathrm{mg}$ de $\mathrm{Cu}^{2+} / \mathrm{g}$ de zeolita sintética después de un tiempo de contacto de 8 horas. El proceso de adsorción de $\mathrm{Cu}^{2+}$ en la zeolita Linde tipo $\mathrm{F}$ fue de acuerdo a la isoterma de Freundlich y es descrito mejor por el modelo cinético de Blanchard de pseudo-segundo orden.

Considerando que existen considerables reservas de roca volcánica (sillar) disponibles, la temperatura relativamente moderada para producir zeolita Linde $\mathrm{F}\left(150^{\circ} \mathrm{C}\right)$, así como la posibilidad de emplear tamaños de partícula 2 y 4 mm., hacen muy viable su conversión con gran potencial de aplicación en diferentes sectores productivos.

\section{REFERENCIAS}

1. Jain, A., Singh, S., Rao, K., Kumar, N., and Tomar, R., 2018, Synthesis and Characterization of Zeolite Linde Type L and its Application in Organic Synthesis, International Journal of Current Researchs, 10(04), 68354 - 683609. https://www.journalcra.com/article/synthesis-and-characterization-zeolite-linde-type-l-and-its-application-organic-synthesis

2. Iftitahiyah, V., Prasetyoko, D., Nur, H., Bahruji, H., 2018, Synthesis an characterization of Zeolite NaX from Bangka Belitung Kaolin as alternative precursor, Malaysian Journal of Fundamental and Applied Sciences, $14(4), 414 \quad-418$. https://core.ac.uk/download/pdf/2877441444.pdf

3. Harja, M., Cimpeanu, S., Dirja, M., and Bucur, D., 2016, Synthesis of Zeolite from Fly Ash and their Use as Soil Amendment, Intech Open Science, chap. 3, 43 - 66. https://www.intechopen.com/books/zeolites-useful-minerals/synthesis-of-zeolite-from-fly-ash-andtheir-use-as-soil-amendment.

4. Ren, X., Qu, R., Liu, S., Zhao, H., Wu, W., Song, H., Zheng, Ch., Wu, X., and Gao, X., 2020, Synthesis of Zeolites from Coal Fly Ash for the Removal of Harmful Gaseous Pollutants: A Review, Aerosol and Air Quality Research, 20, 1127-1144. DOI: https://doi.org/10.4209/aaqr.2019.12.0651

5. Novembre, D., Gimeno, D., and Poe, B., 2019, Synthesis and Characterization of Leucite using a Diatomite Precursor, Scientific Reports, 9(10051), 1 - 10. DOI: https://doi.org/10.1038/s41598-019-46569-y

6. Agulanna, A., Asadu, Ch., and Abuh, M., 2018, Synthesis of zeolite by Thermal Treatment using locally sourced Ugwaka clay (black clay), Journal of materials Science Research and Reviews, 1(2), 1 - 12. http://www.sciencedomain.org/review-history/26143

7. Kyziol-Komosinnska, J., Rosik-Dulewska, C., Franus, M., Antoszczyszyn-Szpicka P., Czupioph, J., Krzyzewska, I., Sorption Capacities of Natural and Synthetic Zeolites for Cu (II) Ions, 2015, Pol. J. Environ. Stud., 24(3), 1111 - 1123. DOI: https://doi.org/10.15244/pjoes/30923

8. Singh, Ah., Jain, A., Rao, K., Kumar, N., and Tomar, R., 2018, Synthesis an Characterization of Zeolite Linde W and its Application as Adsorbent for Malathion, International Journal of Current Research, 11, $75472 \quad-\quad 75476 . \quad$ DOI: https://doi.org/10.24941/ijcr.33245.11.2018 
9. Reis, S., Logli, M. A.,Oliveira, N., 2014, Synthesis and Characterization of Zeolitic Material Derived from Sugarcane Straw Ash, American Journal of Environmental Protection, 2(1), 16 - 21. DOI: https://doi.org/10.12691/env-2-1-4

10. Bhagwanjee, J., and Singh, D. N., 2011, A Review on Synthesis, Characterization and Industrial Applications of Flyash Zeolites, Journal of Materials Education, 33, 65 - 132. https://www.academia.edu/21659710/A_REVIEW_ON_SYNTHESIS_CHARACTERIZATION_AND_INDUSTRIAL_APPLICATIO NS_OF_FLYASH_ZEOLITES

11. Ferrarini, S., Cardoso, A. M., Paprocki, A., and Pires, M., 2016, Integrated Synthesis of Zeolites using Coal Fly Ash: Element distribution in the Products, Washing Waters and Effluent, Braz. Chem. Soc., 27(11), 2034 - $2045 . \quad$ DOI: http://dx.doi.org/10.5935/0103-5053.20160093

12. Miyaji, F., Murakami, T., and Suyana, Y., 2009, Formation of linde F zeolite by KOH treatment of coal fly ash, Journal of the Ceramic Society of Japan, 117(5), 619 - 622. DOI: https://doi.org/10.2109/jcersj2.117.619

13. Santiago, O., Walsh, K., Kele, B., Gardener, E., and Chapman, J., 2016, Novel pre-treatment of zeolite materials for the removal of sodium ions: potential materials for coal seam gas co-produced wastewater, Springer Plus, 5(571), 1 - 16. DOI: https://doi.org/10.1186/s40064-016-2174-9

14. Mohammed, A., Abdulrazzak, F., Himdan, T., 2018, Kinetic Study of Adsorption of Azo Dye from Aqueous solutions by Zeolita and Modified Synthetic Zeolite, Journal of Materials and Environmental Science, 9(9), 2652 - 2659. https://www.jmaterenvironsci.com

15. Han, Ch., Chen, Ch., Cheng, T., and Xie, X., 2016, Adsorption of Lead Ions by Linde type F (K) Zeolite, EDP Sciences, 67, 1 - 7. DOI: https://doi.org/10.1051/matecconf/20166707030

16. Hamadi, A., and Nabih, K., 2018, Synthesis of Zeolites Materials using Fly Ash and Oil Shale Ash and their Applications in Removing Heavy Metals from Aqueous Solutions, Journal of Chemistry, 2018, article ID 6207910, 1 - 13. DOI: https://doi.org/10.1155/2018/6207910

17. Chen, Ch., Ting, Ch., Yisu, S., Yuan, T., 2014, Adsorption of Cu (II) from Aqueous Solution on Fly Ash based Linde F (K) zeolite, Iran J. Chem. Chem. Eng., 33(3), 29 - 35. http://www.ijcce.ac.ir/article_11328.html

18. Hu, T., Gao, W., Liu X., Zhang, Y., and Meng, Ch., 2017, Synthesis of zeolites Na-A and Na-X from tablet compressed and calcinated coal fly-ash, Royal Society of Chemistry, 4, 1 - 11. DOI: https://doi.org/10.1098/rsos.170921

19. Chen, C. and Cheng, T., 2013, Application of Avrami Equation to Kinetics Analysis of Fly Ash based Linde F (K) Zeolite, Asian Journal of Chemistry, 25(4), 1811 - 1813. DOI: http://dx.doi.org/10.14233/ajchem.2013.13156.

20. Ting, Ch., Chen, Ch, Rong, T., Cheng-Hui, H., and Yuan, T., 2018, Competitive Adsorption of Cu, Ni, Pb, and Cd from Aqueous Solution Onto Fly Ash-Based Linde F (K) Zeolite, Iran J. Chem. Chem. Eng. Research, 37(1), $61 \quad-72$. http://www.ijcce.ac.ir/article_31971.html

21. Makgabutlane, B., Nthunya, L., Musyoka, N., Dladla, B., Nxumalo, E., and Mhlanga, S., 2020, Microwave-assisted synthesis of coal fly-ash-based zeolites for removal of ammonium from urine, Royal Society of Chemistry, 10, 2416 - 2427. DOI: https://doi.org/10.1039/c9ra10114d

22. Zhang, Q., Lin, B., Hong, J., and Tang-Chand, Ch., 2017, Removal of ammonium and heavy metals by cost-effective zeolite synthesized from waste quartz sand and calcium fluoride sluge, Water Science \& Technology, 75(3), 587 - 597. DOI: https://doi.org/10.2166/wst.2016.508

23. Nyankson, E., Kwame Efavi, J., Yaya, A, Manu, G., Asare K., Daafuor, J., and Yeboah Abrokwah, R., 2018, Synthesis and characterization of zeolite-A and Zn-exchanged zeolite-A based on natural aluminosilicates and their potential applications, Cogent Engineering, 5, 1 - 23. DOI: https://doi.org/10.1080/23311916.2018.1440480 